\title{
Alterações psicológicas e exercício físico em pacientes com artrite reumatóide
}

\author{
Psychological variables and physical exercise in patients with rheumatoid \\ arthritis
}

\author{
A.B. Dario, W. Külkamp, H.C. Faraco, M.S. Gevaerd, S.C. Domenech
}

\begin{abstract}
Este estudo teve por objetivo realizar uma revisão da literatura, buscando elucidar quais as alterações psicológicas mais prevalentes em indivíduos acometidos por Artrite Reumatóide (AR). Pesquisou-se também a respeito do impacto do exercício físico sobre essas variáveis psicológicas, expondo quais protocolos de exercício que estão sendo empregues com a finalidade de melhorar a saúde mental desses pacientes. Apesar dos poucos estudos sobre o tema, pode-se verificar que os transtornos de ansiedade e de depressão apresentam maior prevalência nessa população. Em contraste, poucas evidências foram encontradas quanto ao maior risco de psicose, agressividade e alto nível de estresse. Paralelamente, desde que foi associado à AR pela primeira vez, na década de 50, o exercício físico vem demonstrando bons resultados no tratamento da doença, consolidando-se cada vez mais como ferramenta terapêutica para esses pacientes. Evidências emergem também em relação às alterações benéficas provocadas pelo exercício nas variáveis psicológicas, no entanto, mais estudos são necessários para o fortalecimento destes dados. Isso permitiria aos pacientes com AR tratamentos mais efetivos, com uma base científica sólida, visando a melhoria na qualidade de vida dentro de um aspecto mais amplo, onde a saúde mental desempenha um papel fundamental.

Palavras-chave: artrite reumatóide, variáveis psicológicas, exercício físico
\end{abstract}

ABSTRACT

The purpose of this study was to provide a literature review aiming to clarify the most prevalent psychological changes present in individuals suffering from rheumatoid arthritis (RA), the impact of exercise on such changes, and the exercise protocols commonly adopted to improve these individuals' mental health. The few studies available report anxiety disorders and depression as most prevalent and physical exercise as a significant therapeutic strategy for this population. There is some evidence of the beneficial effects of exercise on those psychological variables providing RA patients with more effective treatments.

Keywords: arthritis rheumatoid, psychological variables, physical exercise

Submetido: 01.12.2009 | Aceite: 19.04.2010

Amabile Borges Dario, Wladymir Külkamp, Monique da Silva Gevaerd e Susana Cristina Domenech. Laboratório de Análises Multisetorial (MULTILAB); Centro de Ciências da Saúde e do Esporte (CEFID); Universidade do Estado de Santa Catarina (UDESC), Brasil.

Henrique Cabral Faraco. Laboratório de Pesquisas em Desempenho Humano (LAPEDH); Centro de Ciências da Saúde e do Esporte (CEFID); Universidade do Estado de Santa Catarina (UDESC), Brasil.

Endereço para correspondência: Monique da Silva Gevaerd, Centro de Ciências da Saúde e do Esporte - CEFID, Universidade do Estado de Santa Catarina - UDESC, Rua Pascoal Simone, 358, Coqueiros - Florianópolis - SC, CEP 88080-350, Brasil

E-mail: multilab.udesc@gmail.com 
A Artrite Reumatóide (AR) é uma doença inflamatória crônica, de origem auto-imune, com etiologia ainda desconhecida, que causa danos progressivos no sistema musculoesquelético (Plasqui, 2008). A sua prevalência é de aproximadamente .5 a $1.0 \%$ na população em geral, com taxa de incidência de duas a três vezes maior em mulheres, principalmente acima de 40 anos (Ottawa Panel, 2004). O início da doença geralmente acontece na fase mais produtiva da vida, entre os 20 e 50 anos, gerando deformações físicas e limitações dolorosas. Essas manifestações prejudicam a realização das atividades profissionais, sociais e de vida diária, aumentando o impacto da doença sobre a qualidade de vida, bem como, sobre a saúde mental dos pacientes, aumentando o risco de mudanças negativas nos parâmetros psicológicos (Salaffi, Carotti, Gasparini, Intorcia, \& Grassi, 2009).

De fato, foi constatado um aumento na ocorrência de algumas desordens na saúde mental em pacientes diagnosticados com AR, com uma frequência bem acima da média da habitualmente encontrada na população em geral (Isik, Koca, Ozturk, \& Mermi, 2007). Porém, atualmente não há consenso na literatura sobre a origem dessas alterações na saúde mental dos pacientes acometidos por essa patologia, sendo que isso poderia ocorrer em razão de sequelas de uma doença incapacitante ou da própria atividade clínica de uma doença inflamatória crônica, como a AR (Costa, Brasil, Papi, \& Azevedo, 2008).

Recentemente foi demonstrado que a evolução da AR está intimamente relacionada com o aumento de ansiedade e depressão (Costa et al., 2008). Na pesquisa de Isik et al. (2007), a prevalência total de desordens de ansiedade, depressão e ambas (ansiedadedepressão) foi de $70.8 \%$ em pacientes com AR e de $7.3 \%$ em indivíduos saudáveis. Já num estudo realizado no Brasil, encontrou-se uma prevalência em torno de $34 \%$ de transtornos depressivos e ansiosos nesses pacientes (Costa et al., 2008).
Uma forma de amenizar estes problemas de ansiedade e depressão em pacientes com AR poderia estar relacionada à prática regular de exercício físico, pois são evidentes os dados da literatura quanto aos benefícios do exercício no controle da depressão e da ansiedade na população em geral (Coyle \& Santiago, 1995; Raglin \& Wilson, 1996). Adicionalmente, a prática de exercício físico parece ser imprescindível para indivíduos com $\mathrm{AR}$, no sentido de aprimorar sua saúde física (capacidade aeróbia, capacidade funcional e força muscular) (Gaudin et al., 2008; Jong \& Vliet Vlieland, 2005; Noreau, Martineau, Roy, \& Belzile, 1995). Porém, existem poucos estudos que evidenciam os benefícios do exercício físico sobre variáveis psicológicas em pacientes com AR.

Diante do exposto, o presente estudo de revisão teve por objetivo verificar quais alterações psicológicas são mais prevalentes em pacientes com AR, bem como descrever o impacto do exercício físico sobre esses parâmetros psicológicos, expondo quais os protocolos de exercício mais empregues. A seleção das publicações foi realizada entre os meses de Maio e Julho de 2009, sendo utilizados os bancos de dados da biblioteca virtual SciELO (http://www.scielo.br) e o banco de dados da PubMed Central (http://www.pubmedcentral.nih.gov). Artigos de outras bases de dados foram eventualmente incluídos, de acordo com sua relevância e abordagem, de modo a fortalecer e aprimorar a discussão. Foram definidas três fases para a análise dos estudos encontrados. Primeiramente, a análise do título, que deveria possuir o termo "artrite reumatóide" ou o respectivo termo no idioma inglês. Em seguida foram analisados os resumos e, por fim o texto completo, para verificação da adequação do conteúdo à temática. A cada etapa os estudos que não corresponderam aos critérios de seleção foram excluídos. Tal como verificamos na figura 1, da totalidade dos artigos encontrados, apenas 46 se encaixaram nos critérios para integrarem a pesquisa. 


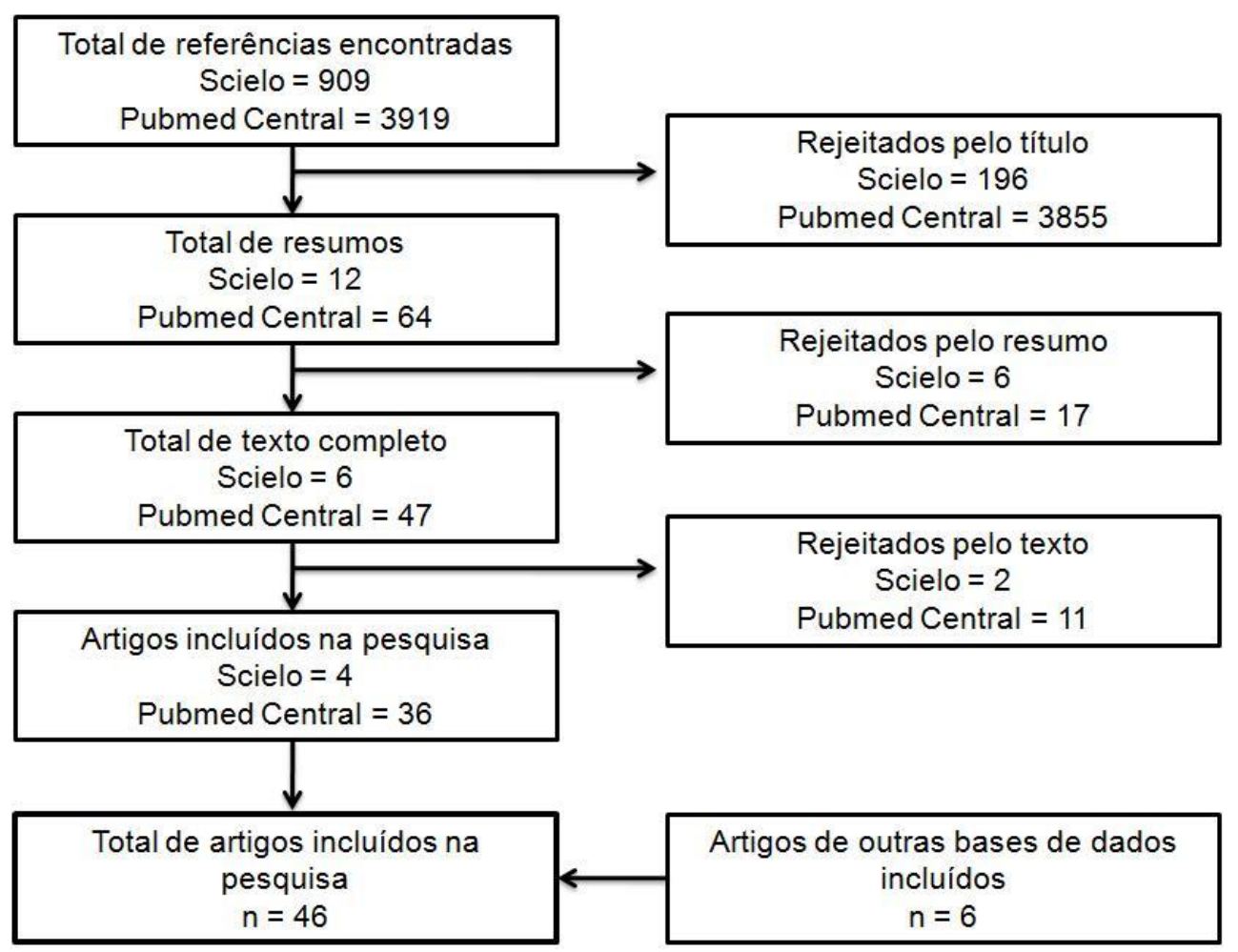

Figura 1. Sumário de inclusão e exclusão dos estudos selecionados para a pesquisa

\section{ARTRITE REUMATÓIDE E SAÚDE MENTAL}

Há mais de 100 anos que a AR figura como um desafio para profissionais da área da saúde, sendo que as formas de prevenção, tratamento e cura não estão totalmente estabelecidas e parecem ser metas sem prazo para serem alcançadas (Külkamp, Dario, Gevaerd \& Domenech, 2009). Atualmente, os principais objetivos do tratamento são diminuição e controle da dor articular, prevenção de perda de função e melhoria da qualidade de vida do paciente (American College of Rheumatology Subcommittee on Rheumatoid Arthritis Guidelines, 2002). Pouco é investigado no âmbito da avaliação e manutenção da saúde mental dos artríticos.

A ocorrência de alterações nos parâmetros psicológicos em indivíduos acometidos por AR foi descrita desde o início do século XX. No final da década de 1950 a doença era interpretada como multifatorial, sendo apontadas, inclusive, causas de caráter emocional (Cormier \& Wittkower, 1957).
Porém, esses achados parecem não ter despertado suficiente atenção no meio científico, o que pode ser evidenciado pela falta de abordagem deste tema em diversos estudos e diretrizes sobre o diagnóstico e o tratamento da AR (American College of Rheumatology Subcommittee on Rheumatoid Arthritis Guidelines, 2002; Bértolo et al., 2007; Costa Pinto, Miguel, \& Resende, 2006; Laurindo et al., 2002; Ottawa Panel, 2004).

Adicionalmente, até o momento não existe consenso na literatura sobre quais alterações psicológicas estão prevalentes em pacientes com AR. Somente alguns manuscritos relatam e evidenciam esses distúrbios psicológicos, isoladamente (Costa et al., 2008; McGowan, 1990; Pincus, Griffith, Pearce, \& Isenberg, 1996; Robinson, Hernandez, Dick, \& Buchanan, 1977; Shih, Hootman, Strine, Chapman, \& Brady, 2006; Sleath et al., 2008).

Segundo Shih et al. (2006), depressão, transtorno de ansiedade generalizada e psicose são graves distúrbios psicológicos, cuja 
prevalência é três vezes maior em portadores de AR, do que em indivíduos sem a doença. Os autores encontraram nesses pacientes associações entre esses distúrbios e dor recorrente, baixo nível sócio-econômico, obesidade, inatividade física, limitações funcionais ou sociais e uma ou mais comorbidades. Além disto, estes distúrbios psicológicos tendem a ser mais prevalentes em mulheres.

Os estudos de Costa et al. (2008), Pincus et al. (1996) e Robinson et al. (1977), também relataram que a depressão e sintomas depressivos são frequentemente encontrados em pacientes com AR, especialmente entre idosos (McGowan, 1990). Porém, Sleath et al. (2008) salientaram que esse tema raramente é abordado, concluindo que a avaliação dos sintomas depressivos deveria ser considerada, especialmente entre aqueles pacientes com menor capacidade funcional.

A prevalência de depressão em indivíduos com AR varia substancialmente, dependendo do instrumento com que os sintomas são mensurados (Martens et al., 2005), podendo variar de 37\% (Suárez-Mendonza, Cardiel, Caballero-Uribe, Ortega-Soto, \& MarquezMarin, 1997) até $66 \%$ (El-Mieddany \& ElRasheed, 2002). Entretanto, evidências sugerem que alguns itens de questionários representam melhor os aspectos de dor e incapacidade do que propriamente a depressão, justificando a discrepância entre valores encontrados (Pincus et al., 1996). Essa prevalência pode também variar de acordo com o gênero. Robinson et al. (1977) verificaram alta prevalência de depressão em pacientes com AR, sendo que mulheres parecem ser mais afetadas do que homens, com percentuais de $60 \%$ e $35 \%$, respectivamente.

Com relação à associação entre depressão e outras variáveis, Robinson et al. (1977) apontaram que em mulheres portadoras de AR os fatores associados foram menor mobilidade nas articulações, diminuição na capacidade funcional e maior dependência, sendo que nenhuma associação foi encontrada entre depressão e dor. No estudo de Costa et al. (2008), foi verificado que o nível de depressão está diretamente relacionado com o nível de atividade da doença, ao contrário dos pacientes em remissão, que não foram diagnosticados com alterações na saúde mental.

Segundo Parker et al. (2003) outras variáveis que podem interferir no quadro emocional, como a fadiga ${ }^{1}$ e perda de energia, estão presentes na rotina dos acometidos por AR. Esses sintomas podem estar relacionados a um maior nível de depressão, o que foi posteriormente confirmado pelo estudo de Pollard, Choy, Gonzalez, Khoshaba e Scott (2006). Outro dado relevante desse último estudo foi a relação entre saúde mental e fadiga, sendo que foi verificado que quanto melhor o nível de saúde mental, menor era o nível de fadiga.

Os transtornos de ansiedade também estão entre as alterações psiquiátricas mais frequentes em pacientes acometidos por doenças crônicas (Grigsby, Anderson, Freedland, Clouse, \& Lustman, 2002; Karajgi, Rifkin, Doddi, \&Kolli, 1990). A prevalência de ansiedade entre os pacientes com AR é alarmante, apresentando-se superior a $70 \%$ em algumas amostras (El-Mieddany \& El-Rasheed, 2002). Num estudo realizado no Brasil a prevalência de ansiedade e depressão foi de $33.7 \%$ dos casos, sendo que os pacientes relataram que essas alterações surgiram somente após o início da doença (Costa et al., 2008).

Estresse e agressividade são distúrbios psicológicos que também podem ser encontrados em pacientes com AR. Muitos portadores da doença convivem com alto nível de estresse, devido à soma do estresse trivial com o estresse provocado pela patologia (McGowan, 1990). Estudos sobre estresse e AR têm mostrado associação entre estes parâmetros, porém sem indicativos de causa-

\footnotetext{
${ }^{1}$ Fadiga: Definida como a sensação de exaustão e diminuição da capacidade para o trabalho físico e mental (NANDA, 2002).
} 
efeito (Wallace, 1987). Cormier e Wittkower, em 1957, comparando irmãos com e sem AR, verificaram que os artríticos apresentavam um quadro de agressividade mais acentuado. Foi recomendado no estudo que os pacientes com distúrbios emocionais fossem avaliados e tratados por psiquiatras, principalmente durante momentos de crises emocionais.

Diante das evidências citadas acima, é importante que os profissionais da área de saúde estejam atentos às alterações psicológicas dos pacientes com AR, pois este diagnóstico influenciará no estabelecimento de metas para o tratamento global do paciente. Adicionalmente, isso pode contribuir para a redução do sofrimento imposto por prejuízos na saúde mental, principalmente a fadiga, por estar mais relacionada a fatores psicossociais do que a marcadores inflamatórios laboratoriais (Riesma et al.,1998).

\section{ALTERAÇõES PSICOLÓGICAS E EXERCÍCIO FÍSICO NA ARTRITE REUMATÓIDE}

Os primeiros estudos relacionando AR e exercício físico foram realizados na década de 1950 e pela primeira vez a prescrição de exercício foi vista como uma forma de tratamento para estes pacientes. Porém, o objetivo dessa intervenção era direcionada à manutenção da amplitude de movimento das articulações (Robinson, 1949).

A tendência do uso do exercício físico como terapêutica para corpo, sem mencionar os benefícios sobre variáveis psicológicas, manteve-se até o início da década de 1980. A partir de então, a terapêutica da AR começou a apresentar uma visão biopsicossocial do paciente, passando a utilizar, além do uso de drogas e cirurgia reconstrutiva, a fisioterapia e o exercício físico, a terapia ocupacional e a educação ou conscientização dos acometidos (Jurisson, 1991; Patterson, 1987; Tucker \& Kirwan, 1991).

Especialmente na última década, um grande número de estudos tem sido conduzido com o intuito de verificar os efeitos do exercício no tratamento de pacientes com AR. Ao contrário do que classicamente foi difundido no meio médico quanto à importância do repouso no tratamento da doença (Baker, 1960; Blair, 1969), estudos indicam que a prática de exercício físico é imprescindível (Bértolo et al., 2007; Gaudin et al., 2008) e que, por meio do mesmo, os portadores de AR podem aprimorar sua saúde (Jong \& Vliet Vlieland, 2005; Mayoux-Benhamou, 2008; Shih et al., 2006).

A prática de exercícios regulares acarreta benefícios psicológicos, tais como: melhor sensação de bem estar, humor e auto-estima, assim como redução da ansiedade, tensão e depressão (Costa, Soares, \& Teixeira, 2007). Já a falta do exercício físico é considerada um fator de risco em pessoas com AR e tem sido associada à depressão e sintomas de depressão (Strine et al., 2004).

Alguns pesquisadores defendem a inclusão incondicional do exercício físico no tratamento da AR (Finckh, Iversen, \& Liang, 2003; Mayoux-Benhamou, 2008), e diversas propostas são apresentadas na literatura especializada e atual que, embora se mostrem divergentes com relação ao tipo de exercício, frequência, duração e intensidade, parecem concordar que o exercício, inclusive protocolos de alta intensidade, são seguros e efetivos no tratamento da doença (Jong \& Vliet Vlieland, 2005; Van den Ende et al., 1996, 2000). No entanto, um estudo que objetivou descrever discussões sobre exercício entre indivíduos com AR e seus reumatologistas, verificou que em somente $53 \%$ das consultas o tema exercício foi abordado, sendo o exercício do tipo aeróbio o mais comentado e apenas $26 \%$ destes pacientes saíram com prescrição para realizar exercícios (Iversen, Eaton \& Daltroy, 2004). É importante observar que esse trabalho revelou haver grande influência do reumatologista sobre a atitude do paciente em realizar exercício, fato que evidencia a necessidade de iniciativa desses profissionais em sugerir a prática de exercício físico como parte integrante da rotina e do tratamento desses pacientes. 


\section{PROTOCOLOS DE EXERCÍCIO FÍSICO}

Ainda hoje a grande maioria dos protocolos está baseada no modelo de prescrição de exercícios para a população saudável (Gaudin et al., 2008), não existindo consenso quanto a um modelo padrão de programas de exercício físico para indivíduos com AR (Külkamp et al., 2009). Embora exista um grande número de estudos experimentais com exercício físico em artríticos, poucos focam nas alterações dos parâmetros psicológicos provocadas pelo exercício. Porém, alguns que tiveram essa pretensão mostraram evidências positivas sobre aspectos psicológicos (ver Tabela 1) (Bilberg, Ahlmen, \& Mannerkorpi, 2005; Eversden, Maggs, Nightingale, \& Jobanputra 2007; Jong et al., 2003).

Numa comparação entre exercício aquático (piscina aquecida a $35^{\circ} \mathrm{C}$ ) e exercício físico em solo em pacientes com AR, usando uma escala visual analógica de 7 pontos para medir o efeito do tratamento sobre o bem estar, foi verificado que imediatamente após completar o tratamento, os indivíduos que realizaram hidroterapia relataram sentir-se melhor ou muito melhor do que os que praticaram atividades em solo. Os protocolos utilizados no estudo eram similares, continham sessões de 30 minutos que iniciavam com aquecimento (mobilização e alongamento), seguido de exercício com foco em mobilidade articular, força muscular $e$ atividades funcionais $e$ finalizavam com atividades de volta à calma. Ambos protocolos tiveram 6 semanas de duração (Eversden et al., 2007).

Outro estudo experimental de 12 semanas de duração concluiu que o exercício aquático em piscina aquecida aumentou o nível de vitalidade, além de aumentar significativamente a resistência muscular das extremidades inferiores e superiores de pacientes com AR. Apesar da melhoria desses parâmetros, a saúde mental, analisada através do SF-36, não apresentou uma alteração significativa. Essa pesquisa seguiu o protocolo de 45 minutos de exercícios aeróbios (70\% FC máxima), resistência muscular (com cadência estabelecida) e coordenação, realizados duas vezes por semana (Bilberg et al., 2005).

Adicionalmente, um estudo verificou o efeito de atividades aeróbias de baixo impacto em pacientes com AR com idade entre 40 e 70 anos, envolvendo três grupos experimentais: (i) programa de exercício orientado; (ii) programa de exercício orientado indirectamente através de fita de vídeo no domicílio; e, (iii) sem prescrição de exercício, controle. Verificou-se que após 12 semanas de execução da atividade os grupos de exercício (i e ii) apresentaram redução nos sintomas de depressão, além de melhorarem os parâmetros de dor e fadiga, sem diferença significativa entre eles. Outros benefícios verificados foram a diminuição do tempo na execução do teste de caminhada de 50 pés (15.24 metros) e o aumento da força no teste de preensão manual, sendo que esses dados representam melhoria na capacidade funcional dos pacientes de ambos os grupos de exercício (Neuberger et al., 2007).

A partir de um estudo experimental com duração de dois anos, que se propôs a verificar a efetividade e a segurança de um programa de exercícios dinâmicos de alta intensidade e longa duração em pacientes com AR (Jong et al., 2003), surgiu o programa RAPIT (Rheumatoid Arthritis Patients In Training). Esse protocolo talvez represente hoje a mais completa tentativa de padronização de avaliação, controle e prescrição de exercício físico no tratamento da AR (Külkamp et al., 2009). Seus autores (Jong et al., 2003; Munneke et al., 2003) propõem exercícios aeróbios, fortalecimento muscular e jogos desportivos coletivos (como vôlei, basquetebol e futsal), totalizando 75 minutos por sessão, realizadas duas vezes por semana. O exercício aeróbio utilizado nesse estudo foi o ciclismo estacionário, realizado em duração e intensidade progressivas. Já para os exercícios de fortalecimento muscular os autores propõem o treino em circuito, com oito a dez exercícios realizados em 8 a 15 repetições e com um intervalo decrescente de 90 para $60 \mathrm{~s}$. 


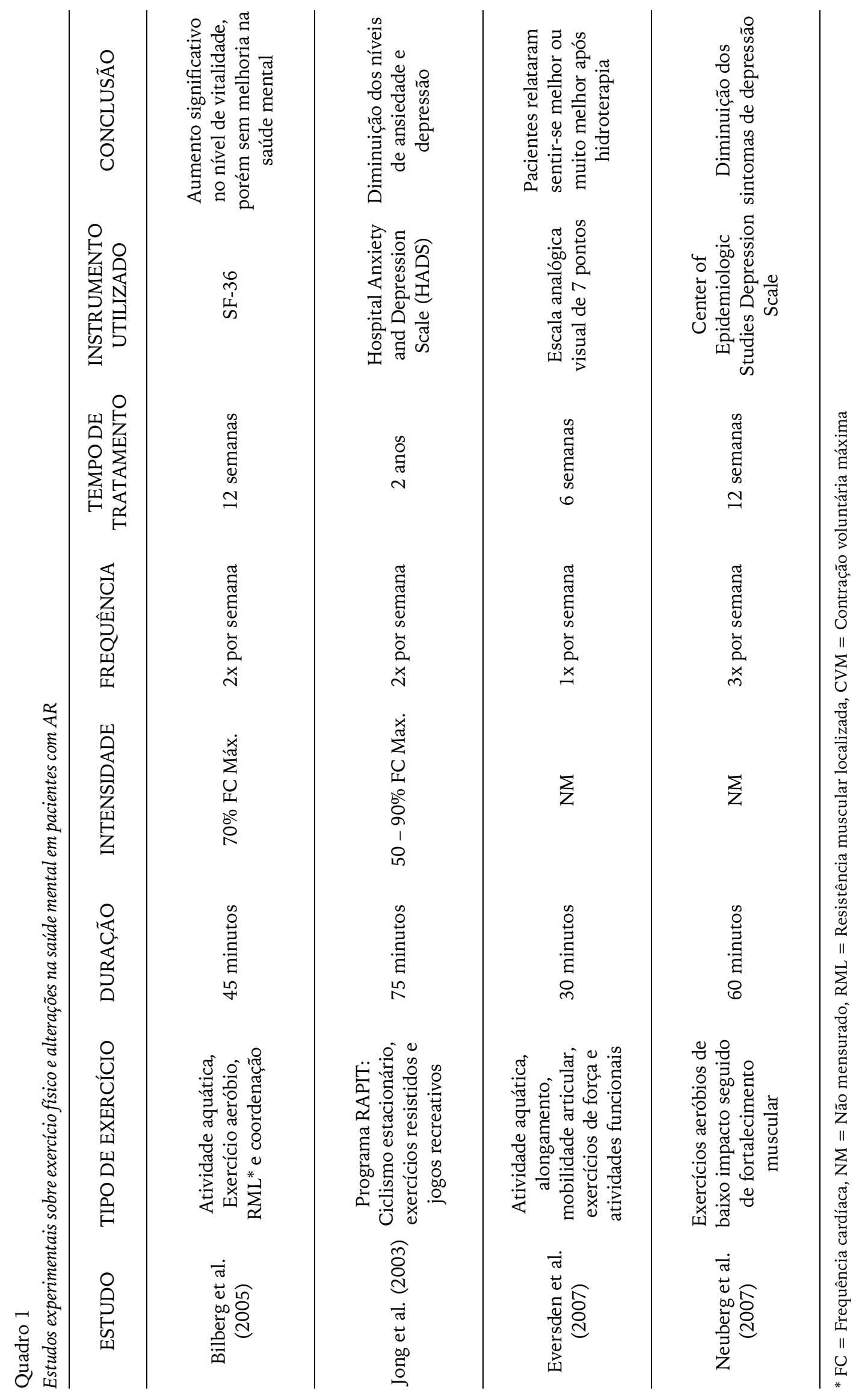


Sugere-se, ainda, que a cada oito semanas a rotina dos exercícios deve ser substituída. Este estudo demonstrou que com a participação em um programa de exercícios de alta intensidade e longa duração, os pacientes com AR melhoram variáveis da saúde mental, como ansiedade e depressão, sem detrimento na atividade da doença (Jong et al., 2003).

Em outra publicação do mesmo grupo de pesquisadores do RAPIT, foi verificado que mesmo depois de 2 anos de exercício dinâmico intenso, a aderência e satisfação dos participantes ao programa foi alta (Munneke et al., 2003).

\section{CONSIDERAÇÕES FINAIS}

Historicamente, a AR é interpretada como uma condição clínica potencialmente grave, crônica e incapacitante, que acarreta alterações no âmbito físico e emocional dos pacientes. Apesar dos poucos estudos relacionados às alterações psicológicas desses indivíduos, parece haver um consenso em relação à maior prevalência de transtornos de ansiedade e depressão nessa população. Poucas evidências foram encontradas quanto ao maior risco de psicose, agressividade e alto nível de estresse nesses indivíduos.

Sobre o exercício físico e sua inclusão como parte do tratamento, apesar de já mencionado na metade do século XX, ainda percebe-se a necessidade de maior iniciativa por parte dos reumatologistas em incentivar a prática de exercícios físicos aos seus pacientes. Tal necessidade é evidenciada pela idéia do exercício como auxiliar terapêutico, podendo promover alterações positivas nas variáveis de saúde mental e com impacto direto na qualidade de vida.

Atualmente, não existe um protocolo de exercício mais recomendado para o tratamento da AR, muito menos um modelo focado no aprimoramento dos parâmetros psicológicos. Apesar disso, tem sido verificado que o exercício físico aquático parece ser bem aceite e apresenta bons resultados nessa população. Contudo, vale ressaltar que mais importante do que determinar qual o melhor tipo ou qual a intensidade ideal de exercício para esses indivíduos, é considerar que a doença apresenta-se de forma cíclica, em períodos caracterizados como ativo e de remissão, e que o protocolo de exercício deverá ser proposto de acordo com o estado geral do indivíduo e não apenas com base em normatizações ou exames clínicos. Além disso, os profissionais da área da saúde devem estar cientes que os portadores de AR podem apresentar momentos de crises emocionais e um profissional responsável nessa área deve estar presente para auxiliá-los.

Embora haja um número limitado de estudos que utilizaram o exercício físico como intervenção visando melhoria de variáveis psicológicas, evidências emergem em relação às alterações benéficas provocadas pelo exercício físico nessas variáveis. Todavia, para que essa proposta terapêutica de aprimoramento na saúde mental através do exercício seja incorporada na rotina clínica dos pacientes, mais estudos devem ser realizados de modo a confirmar criteriosamente essas evidências e possibilitar a utilização desse recurso terapêutico sem efeitos colaterais indesejáveis aos pacientes com AR.

\section{REFERÊNCIAS}

American College of Rheumatology - Subcommittee on Rheumatoid Arthritis Guidelines (2002). Guidelines for the management of rheumatoid arthritis: 2002 update. Arthritis and Rheumatism, $46,328-346$.

Baker F. (1960). Rheumatoid arthritis: Present-day physical therapy. California Medicine, 92, 330333.

Bértolo, M. B., Brenol, C. V., Schainberg, C. G., Neubarth, F., Lima, F. A. C., Laurindo, I. M., et al. (2007). Atualização do consenso brasileiro no diagnóstico e tratamento da artrite reumatóide. Revista Brasileira de Reumatologia, 47, 151-159.

Bilberg, A., Ahlmen, M., \& Mannerkorpi, K. (2005). Moderately intensive exercise in a temperate pool for patients with rheumatoid arthritis: A randomized controlled study. Rheumatology, 44, 502-508. 
Blair, D. C. (1969). Physical treatment for rheumatoid arthritis and other amenable conditions. Canadian Family Physician, 15, 40-42.

Cormier, B. M., \& Wittkower, E. D. (1957). Psychology and rheumatoid arthritis. Canadian Medical Association Journal, 77, 533-541.

Costa Pinto, M. R., Miguel, R. C. C., \& Resende, G. G. (2006). Tratamento da artrite reumatóide. Revista Brasileira de Reumatologia, 46 (1), 219-223.

Costa, A. F. C., Brasil, M. A. A., Papi, J. A., \& Azevedo, M. N. L. (2008). Depressão, ansiedade e atividade de doença na artrite reumatóide. Revista Brasileira de Reumatologia, 48(1), 7-11.

Costa, R. C., Soares, H. R. L., \& Teixeira, J. A. C. (2007). Benefícios da atividade física e do exercício físico na depressão. Revista do Departamento de Psicologia - UFF, 19(1), 269-276.

Coyle, C. P., \& Santiago, M. C. (1995) Aerobic exercise training and depressive symptomatology in adults with physical disability. Archives of Physical Medicine and Rehabilitation, 76, 647-652.

El-Mieddany, Y. M., \& El-Rasheed, A. H. (2002). Is anxiety a more common disorder than depression in rheumatoid arthritis? Joint Bone Spine, 69, 300-306.

Eversden, L., Maggs, F., Nightingale, P., \& Jobanputra, P. (2007). A pragmatic randomized controlled trial of hydrotherapy and land exercises on overall well being and quality of life in rheumatoid arthritis. BioMed Central Musculoskeletal Disorders, 8. Consultado em 12 de Outubro de 2009 a partir de http//www.biomedcentral.com/1471-1474.

Finckh, A., Iversen, M., \& Liang, M. H. (2003). The exercise prescription in rheumatoid arthritis: Primum non nocere. Arthritis and Rheumatism, 48, 2393-2395.

Gaudin, P., Leguen-Guegan, S., Allenet, B., Baillet, A., Grange, L., \& Juvin, R. (2008). Is dynamic exercise beneficial in patients with rheumatoid arthritis? Joint Bone Spine, 75, 11-17.

Grigsby, A. B., Anderson, R. J., Freedland, K. E., Clouse, R. E., \& Lustman, P. J. (2002). Prevalence of anxiety in adults with diabetes: A systematic review. Journal of Psychosomatic Research, 53, 1053-1060.

Isik, A., Koca, S. S., Ozturk, A., \& Mermi, O. (2007). Anxiety and depression in patients with rheumatoid arthritis. Clinical Rheumatology, 26(6), 872-878.
Iversen, M. D., Eaton, H. M., \& Daltroy, L. H. (2004). How rheumatologists and patients with rheumatoid arthritis discuss exercise and the influence of discussions on exercise prescriptions. Arthritis and Rheumatism, 15, 6372.

Jong, Z., \& Vliet Vlieland, T. P. M. (2005). Safety of exercise in patients with rheumatoid arthritis. Current Opinion in Rheumatology, 17, 177-182.

Jong, Z., Munneke, M., Zwinderman, A. H., Kroon, H. M., Jansen, A., Ronday, K. H., et al. (2003). Is a long-term high-intensive exercise program effective and safe in patients with rheumatoid arthritis? Arthritis and Rheumatism, 48(9), 24152424.

Jurisson, M. L. (1991). Rehabilitation in rheumatic diseases: What's new. Western Journal of Medicine, 154, 545-548.

Karajgi, B., Rifkin, A., Doddi, S., \& Kolli, R. (1990). The prevalence of anxiety disorders in patients with chronic obstructive pulmonary disease. American Journal of Psychiatry, 147, 200-201.

Külkamp, W., Dario, A. B., Gevaerd, M. S., \& Domenech, S. C. (2009). Artrite reumatóide e exercício físico: Resgate histórico e cenário atual. Revista Brasileira de Atividade Física e Saúde, 14, 51-60.

Laurindo, I. M. M., Pinheiro, G. R. C., Ximenes, A. C., Xavier, R. M., Giorgi, R. D. N., Ciconelli, R. M., et al. (2002). Consenso brasileiro para diagnóstico e tratamento da artrite reumatóide. Revista Brasileira de Reumatologia, 42, 355-361.

Martens, M. P., Vandyke, M., Parker, J. C., Smarr, K. L., Hewett, J. E., Hewett, J. E., \& Walker, S. E. (2005). Analyzing reliability of change in depression among persons with rheumatoid arthritis. Arthritis and Rheumatism, 53, 973-978.

Mayoux-Benhamou, A. (2008). Get moving! Dynamic exercise therapy for rheumatoid arthritis. Joint Bone Spine, 75, 3-4.

McGowan, P. (1990). Psychologic, social, emotional, and practical problems of patients with arthritis. Canadian Family Physician, 36, 503-507.

Munneke, M., Jong, Z., Zwinderman, A. H., Jansen, A., Ronday, H. K., Peter, W. F. H., et al. (2003). Adherence and satisfaction of rheumatoid arthritis patients with a long-term intensive dynamic exercise program (RAPIT program). Arthritis and Rheumatism, 49, 665-672.

Neuberger, G. B., Aaronson, L. S., Gajewski, B., Embretson, S. E., Cagle, P. E., Loudon, J. K., \& Miller, P. A. (2007). Predictors of exercise and 
effects of exercise on symptoms, function, aerobic fitness and disease outcomes of rheumatoid arthritis. Arthritis and Rheumatism, 57, 943-952.

Noreau, L., Martineau, H., Roy, L., \& Belzile, M. (1995). Effects of a modified dance-based exercise on cardiorespiratory fitness, psychological state and health status of persons with rheumatoid arthritis. American Journal of Physical Medicine \& Rehabilitation, 74, 19-27.

North American Nursing Diagnosis Association NANDA (2002). Diagnóstico de enfermagem da NANDA: Definições e classificações 2001-2002. Porto Alegre: Artmed.

Ottawa Panel (2004): Evidence-based clinical practice guidelines for therapeutic exercises in the management of rheumatoid arthritis in adults. Physical Therapy, 84, 934-972.

Parker, J. C., Smarr, K. L., Slaughter, J. R., Johnston, S. K., Priesmeyer, M. L., Hanson, K. D., et al. (2003). Management of depression in rheumatoid arthritis: A combined pharmacologic and cognitive-behavioral approach. Arthritis and Rheumatism, 49, 766-777.

Patterson, A. C. (1987). Update on treatment of rheumatoid arthritis. Canadian Family Physician, 33, 665-670.

Pincus, T., Griffith, J., Pearce, S., \& Isenberg, D. (1996). Prevalence of self-reported depression in patients with rheumatoid arthritis. British Journal of Rheumatology, 35, 879-883.

Plasqui, G. (2008). The role of physical activity in rheumatoid arthritis. Physiology \& Behavior, 94, 270-275.

Pollard, L. C., Choy, E. H., Gonzalez, J., Khoshaba, B., \& Scott, D. L. (2006). Fatigue in rheumatoid arthritis reflects pain, not disease activity. Rheumatology, 45, 885-889.

Raglin, J. S., \& Wilson, M. (1996). State anxiety following 20 minutes of bicycle ergometer exercise at selected intensities. International Journal of Sports Medicine, 17, 467-471.

Riesma, R. P., Rasker, J. J., Taal, E., Griep, E. N., Wouters, J. M. G., \& Wiegman, O. (1998). Fatigue in rheumatoid arthritis: The role of self-efficacy and problematic social support. British Journal of Rheumatology, 37, 1042-1046.

Robinson, D. (1949). Rheumatoid arthritis. Canadian Medical Association Journal, 61, 152-156.

Robinson, E. T., Hernandez, L. A., Dick, W. C., \& Buchanan, W. W. (1977). Depression in rheumatoid arthritis. Journal of the Royal College of General Practitioners, 27, 423-427.

Salaffi, F., Carotti, M., Gasparini, S., Intorcia, M., \& Grassi, W. (2009). The health-related quality of life in rheumatoid arthritis, ankylosing spondylitis, and psoriatic arthritis: A comparison with a selected sample of healthy people. Health and Quality of Life Outcomes, 7, 2537.

Shih, M., Hootman, J. M., Strine, T., Chapman, D. P., \& Brady, T. J. (2006) Serious psychological distress in U.S. adults with arthritis. Journal of General Internal Medicine, 21, 1160-1166.

Sleath, B., Chewning, B., Vellis, B. M., Weinberger, M., Vellis, R. F., Tudor, G., \& Beard, A. (2008). Communication about depression during rheumatoid arthritis patients visits. Arthritis and Rheumatism, 59, 186-191.

Strine, T. W., Hootman, J. M., Okoro, C. A., Balluz, L., Moriarty, D. G., Owens, M., \& Mokdad, A. (2004). Frequent mental distress status among adults with arthritis age 45 years and older, 2001. Arthritis and Rheumatism, 51, 533-537.

Suárez-Mendonza, A. A., Cardiel, M. H., CaballeroUribe, C. V., Ortega-Soto, H. A., \& MarquezMarin, M. (1997). Measurement of depression in Mexican patients with rheumatoid arthritis: Validity of the Beck Depression Inventory. Arthritis and Rheumatism, 10, 194-199.

Tucker, M., \& Kirwan, J. R. (1991). Does patient education in rheumatoid arthritis have therapeutic potential? Annals of the Rheumatic Diseases, 50, 422-428.

Van den Ende, C. H., Breedveld, F. C., Cessie, S. L., Dijkmans, B. A. C., de Mug, A. W., \& Hazes, J. M. W. (2000). Effect of intensive exercise on patients with active rheumatoid arthritis: A randomized clinical trial. Annals of the Rheumatic Diseases, 59, 615-621.

Van den Ende, C. H., Hazes, J. M., Cessie, S., Mulder, W. J., Belfor, D. G., Breedveld, F. C., \& Dijkmans, B. A. (1996). Comparison of high and low intensity training in well controlled rheumatoid arthritis: Results of a randomized clinical trial. Annals of the Rheumatic Diseases, 55, 798-805.

Wallace, D. J. (1987). The role of stress and trauma in rheumatoid arthritis and systemic lupus erythematosus. Seminars in Arthritis and Rheumatism, 16, 153-157. 